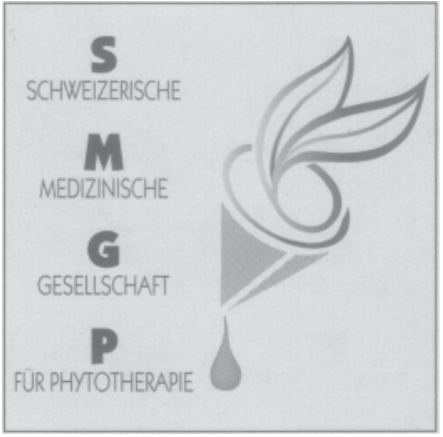

\section{Jahrestagung Phytotherapie diskutiert evaluative Forschung}

Diskussionen um die Wirksamkeit pflanzlicher Arzneimittel und der Phytotherapie insgesamt flammen immer wieder auf. Die $z u$ erwartenden Resultate des sich vor dem Abschluss befindenden Projektes Evaluation Komplementärmedizin (PEK), das vor 5 Jahren vom Bundesamt für Sozialversicherung lanciert wurde, veranlassten die Schweizerische Medizinische Gesellschaft für Phytotherapie (SMGP), sich der wissenschaftichen Diskussion zu stellen. Der Wagemut zahlte sich aus: Die Phytotherapie erhielt von unabhängigen Forschern gute Noten. Die Phytotherapeuten selbst bewiesen mit klar strukturierten Überlegungen die Vorteile und die Notwendigkeit einer an Arzneipflanzen orientierten Medikation in verschiedenster Hinsicht.

300 Teilnehmerinnen und Teilnehmer nahmen an der Tagung zum Thema «Evaluation Phytotherapie - eine Bilanz» teil und dokumentierten damit ihr Interesse an dieser Therapierichtung. Die Phytotherapie steht derzeit, so Tagungsleiter Prof. Dr. Beat Meier, in einer widersprüchlichen Situation: Einerseits machen sowohl die Ärzteund Apothekerschaft wie auch die Patientinnen und Patienten tagtäglich positive Erfahrungen mit pflanzlichen Zubereitungen, andererseits wird teilweise auf behördlich-politischer, insbesondere jedoch auf naturwissenschaftlich-universitärer Ebene die Evidenz von Phytotherapeutika in Frage gestellt. Dass jedoch die Phytotherapie die naturwissenschaftliche Herausforderung nicht scheuen muss, bewiesen die nachfolgend in Auszügen vorgestellten Referate.

\section{Studien- versus Praxissituation}

Professor Dr. med. Volker Schulz, Arzt für Innere Medizin, Berlin, befasste sich in seinem Referat mit dem Nutzen, den Risiken und Kosten von pflanzlichen Arzneimitteln am Beispiel von Ginkgo biloba. Dabei be-

\section{Gesellschaftsmitteilungen · Society Bulletins}

Forsch Komplementärmed Klass Naturheilkd 2005;12:121-122 leuchtete er vor allem die Anforderungen, die Bewertung und die Aussagekraft von klinischen Studien. Generell gelten die statistischen Gruppendifferenzen zwischen Verum und Plazebo als «Goldstandard», auf dessen Grundlage die «Evidenz» zukünftiger Behandlungen beurteilt wird. Mit solchen klinischen Doppelblindstudien werden jedoch Therapien in einem artifiziellen Umfeld geprüft, das oft nicht die Situation in der Praxis repräsentiert. Diese einseitige Orientierung, die nur an der Pharmakodynamik der Wirkstoffe ausgerichtet ist, vernachlässigt den Beitrag, den das «therapeutische Umfeld» auf den Behandlungserfolg haben kann. Diese Situation hat, so Schulz, in zurückliegenden Jahrzehnten zu aufwendigen Neuentwicklungen von Arzneimitteln geführt, die sich für die Praxis als nicht notwendig erwiesen oder die im praktischen Einsatz versagt haben.

\section{Ginkgo wirksam und kostengünstig}

Als aktuelles Beispiel verglich Schulz die synthetisch hergestellten Acetylcholinesterase-(CHE-)Hemmstoffe mit Ginkgo-biloba-Spezialextrakten (EGb) bei der Behandlung der Demenz vom Alzheimer-Typ (DAT) sowie der vaskulär bedingten Demenz. Für EGb und für CHE-Hemmer liegen Studien von - bezogen auf das Krankheitsgeschehen - mittelfristiger Dauer (24-56 Wochen) zum Nachweis der Wirksamkeit gemäss den gültigen Leitlinien der EU vor. Betrachtet man die Ergebnisse für die international anerkannte und als primäre Zielgrösse verwendete «Alzheimer's Disease Assessment Scale» (ADAS-cog) sind zwischen den Medikationen keine signifikanten Unterschiede erkennbar. Eine relativ geringe numerische Differenz zugunsten der CHE-Hemmer kann als Folge von spezifischen Nebenwirkungen (initial Brechreiz und Erbrechen in einer Mehrzahl der Fälle) mit sukzessiver Entblindung der Gruppenzugehörigkeit interpretiert werden. Die Häufigkeit unerwünschter Arzneimittelwirkungen liegt bei
Ginkgo mit 1-2\% um mehr als eine Zehnerpotenz niedriger als bei den CHE-Hemmern. Die Behandlungskosten pro Tag betragen bei optimaler Dosierung bei den CHE-Hemmern das 3- bis 6-fache derjenigen mit dem herkömmlichen GinkgoExtrakt. In absoluten Beträgen würde dies pro Patient einem jährlichen Kostenunterschied von etwa $1000 €$ entsprechen. Eine konsequente Umstellung von etwa einer Million Behandlungsbedürftigen in Deutschland auf EGb würde eine jährliche Kosteneinsparung von einer Milliarde $€$ zur Folge haben.

Da die Verdrängung traditioneller durch neue synthetische Arzneimittel mit hohen wirtschaftlichen Risiken verbunden sein kann, plädierte Schulz dafür, dass die klinische Forschung sich mehr als bisher dem Studium von bereits vorhandenen, insbesondere pflanzlichen Arzneimitteln widmet. Allein auf die Pharmakodynamik der Wirkstoffe zu fokussieren, reicht nicht aus. Es sollten bei geeigneten Indikationen diejenigen Prüfverfahren anerkannt und gegebenenfalls bevorzugt werden, welche die Gesamtheit der Therapieerfolge unter den Gegebenheiten der Praxis abbilden.

\section{Gute Noten durch Patienten für ärztliche}

\section{Phytotherapie}

Im Rahmen des PEK wurde eine Feldstudie durchgeführt, die im ersten Teil die Struktur von konventionellen und komplementärmedizinischen Praxen erfasste und verglich. In der zweiten Phase wurde das Patientenprofil im komplementärmedizinischen und im konventionellen Bereich anhand der Antworten auf eine Patientenumfrage eruiert. 365 Arztpraxen aus der ganzen Schweiz beteiligen sich an diesem Programm. Ausgewertet wurden zirka 20000 Konsultationen (weitere Informationen zur Gesamtstudie auf www.pekswiss.ch). Der Anteil der phytotherapeutisch orientierten Ärzte war zwar sehr klein, zeigte aber doch interessante Resultate, die Projektleiter PD Dr. André Busato, Universität Bern, vorstellte. Dem- 
nach entspricht die Versorgungsstruktur und die Demographie der Patientenpopulation der Phytotherapeuten derjenigen anderer komplementärmedizinischer Fachrichtungen. Diese Ärzte behandeln eher chronische Krankheiten und Patienten mit schweren Problemen, wobei die höhere Morbidität jedoch nicht zu einer vermehrten Inanspruchnahme von Leistungen führt. Psychische Krankheiten und Probleme des Bewegungsapparates bilden die wichtigsten Indikationen. Phytotherapie wird meistens mit anderen Therapien kombiniert und nur selten als Monotherapie eingesetzt. Die Lebensqualität der Patienten unterscheidet sich nicht von konventionell behandelten Patienten, ihre Zufriedenheit ist jedoch konsistent höher. Die Kommunikation Arzt/Patient, die Motivation und teilweise die medizinisch-technische Kompetenz der Ärztinnen und Ärzte werden besser beurteilt als in der Schulmedizin. Die ärztlichen Phytotherapeuten erhalten also durch diese Studie gute Noten und arbeiten mit hoher Fachkompetenz.

\section{Phytotherapeutika in der evaluativen Forschung: Äquivalent zu synthetischen Arzneimitteln}

Im Rahmen der Literaturstudie des PEK wurden phytotherapeutische und vergleichbare Studien mit synthetischen Arzneimitteln analysiert. Aus einer umfassenden Literatursuche in 19 elektronischen Datenbanken wurde aus einer Gesamtheit von 986 (eine bemerkenswerte Zahl) publizierten klinischen Studien mit Arzneipflanzen eine zufallsgenerierte Stichprobe von 237 Publikationen ausgewählt. Davon konnten 89 plazebokontrollierte phytotherapeutische Studien der traditionellen europäischen Phytotherapie zugeordnet werden. Sie wurden 89 Studien aus der konventionellen Medizin mit vergleichbaren Indikationen und Endpunkten, die im Cochrane Controlled Trials Register (CCTR, Ausgabe I, 2003) verzeichnet waren, gegenübergestellt. Alle Studien wurden von zwei Experten einer unabhängigen Beurteilung unterzogen. Der Effekt von systematischen Verzerrungen wurde mittels graphischen (Funnel-Plots) und statistischen Methoden (stratifizierte Metaanalysen, Random-effects-Metaregressionsmodelle) untersucht. Peter Jüni, Institut für Sozial- und Präventivmedizin, Universität Bern, stellte die Resultate vor. Die Studien aus der Phytotherapie waren kleiner, sie wurden weniger häufig in englischsprachigen Zeitschriften veröffentlicht, und sie waren weniger häufig in Medline indexiert als die untersuchten Studien der konventionellen Medi- zin. 19 Studien (21\%) der Phytotherapie gegenüber nur 4 Studien (5\%) der konventionellen Medizin waren von höherer methodologischer Qualität. Wobei zu beachten ist, dass die Beurteilung ausschliesslich auf Grund der Publikationen und nicht nach der Konsultation der Rohdaten erfolgte. Aus Platzgründen werden wichtige Parameter für Metaanalysen nicht oft vollständig wiedergegeben. Systematische Verzerrungen finden sich sowohl in plazebokontrollierten Studien der Phytotherapie wie auch in der konventionellen Medizin. Nach der Korrektur dieser verzerrenden Faktoren zeigen sowohl Phytotherapie wie auch die konventionelle Medizin insgesamt Resultate, die statistisch gesichert über den Plazeboeffekt hinausgehen. Die therapeutischen Effekte fallen zudem tendenziell eindeutiger zugunsten der Phytotherapie aus. Insgesamt ist - was in Anbetracht vieler Kommentare zu pflanzlichen Arzneimitteln, die vorliegende Studien kritisieren, auch den Referenten überraschte - die Qualität der phytotherapeutischen Studien besser als die Qualität der Studien mit synthetischen Arzneimitteln. Man darf gespannt auf die Reaktionen auf diese Studie in der internationalen Fachwelt warten, wenn sie wie geplant in einem renommierten Journal veröffentlicht sein wird. Ob sie es schafft, die zu beobachtende Skepsis gegenüber Studien mit pflanzlichen Arzneimitteln schwinden zu lassen und die Diskussion um deren Wirksamkeit auf eine positive Ebene zu verlegen?

\section{Inkonsistente Resultate führen zu Interpretationsproblemen}

Randomisierte Studien der letzten Jahrzehnte im Bereich der Phytotherapie führten zum Teil zu unterschiedlichen und gelegentlich widersprüchlichen Ergebnissen. So zeigte PD Dr. med. Klaus Linde, Zentrum für naturheilkundliche Forschung, Technische Universität München, dass einzelne Studien deutliche Effekte von SonnenhutExtrakten bei der Frühbehandlung von Erkältungskrankheiten gegenüber Plazebo, andere dagegen keinerlei Effekt aufwiesen. Hier ist die grosse Heterogenität der getesteten Präparate (unterschiedliche Echinacea-Spezies, Pflanzenteile, Extraktionsverfahren, Standardisierungen, Dosierungen, galenische Formen) von Bedeutung. Festzuhalten ist, dass die in der Schweiz und Deutschland seit Jahrzehnten zugelassenen, wichtigsten Präparate zu denjenigen zählen, die die erwarteten Effekte zeigten. Bei Studien mit Johanniskrautextrakten fällt auf, dass europäische Studien zur Behandlung leichter bis mittelschwerer De- pressionen meist eine Überlegenheit von Verum gegenüber Plazebo zeigen, während neuere, vor allem amerikanische Studien lediglich bescheidene oder keine Effekte erkennen liessen. Hier dürfte nicht die Unterschiedlichkeit der geprüften Präparate, sondern die Heterogenität des Patientenkollektivs sowie eine unterschiedliche Auffassung des Krankheitsbildes eine wesentliche Rolle spielen. Während in die europäischen Studien meist ein in der niedergelassenen Praxis häufiger Mix aus Patienten mit eher milderen depressiven Störungen eingeschlossen wurde, sind die amerikanischen Studien fast ausnahmslos auf $\mathrm{Pa}$ tienten beschränkt, welche die Diagnosekriterien einer «Major Depression» erfüllen. Die Metaregression deutet darauf hin, dass dies einen Einfluss hat, d.h. dass Johanniskrautextrakte bei tendenziell stärker ausgeprägten, majoren Depressionen weniger wirksam sind als bei leichteren depressiven Störungen. Dies entspricht den Erfahrungen, die in der europäischen Phytotherapie schon von der Kommission E niedergelegt wurden. Interessanterweise sind in den 14 Studien, in denen unterschiedliche Johanniskrautpräparate mit synthetischen Antidepressiva verglichen wurden, die Ergebnisse homogen. Nach diesen Untersuchungen waren die untersuchten Johanniskrautextrakte ähnlich wirksam wie ältere Antidepressiva und selektive Serotonin-Wiederaufnahmehemmer. Selbstverständlich ist davon auszugehen, dass auch bei Studien von synthetischen Arzneimitteln oder nichtmedikamentösen Interventionen widersprüchliche Ergebnisse auftreten. Nach Erfahrung des Referenten im Bereich der Migräneprophylaxe sind sie jedoch weniger ausgeprägt als im Beispiel von Echinacea, das nach Meinung der Berichterstatter innerhalb der Phytotherapie in seiner Komplexität als Spezialfall zu betrachten ist.

\section{(Fortsetzung Heft 3/05)}

\section{Beatrix Falch, Beat Meier}

Korrespondenzadresse:

Geschäftsstelle SMGP

Prof. Dr. Beat Meier

c/o Hochschule Wädenswil

Grüental, Postfach 335

8820 Wädenswil, Schweiz

E-mail b.meier@hsw.ch

Weitere Informationen zur Jahrestagung und zur Schweizerischen Medizinischen Gesellschaft für Phytotherapie und zum Ausbildungsangebot in Phytotherapie unter www.smgp.ch 